\title{
RENDIMIENTO DIAGNÓSTICO DEL CULTIVO Y SUSCEPTIBILIDAD DE Helicobacter pylori EN PACIENTES PERUANOS: RESULTADOS DE UN LABORATORIO CENTINELA
}

\author{
Maricruz Olano®1,2,a, Manuel Chu(1,2,b, Jesús Guzmán(11,2,c, Denis Castillo®1,2, b, \\ Michel Sauvain $11,2, \mathrm{~d}$ \\ 1 Laboratorio Mixto Internacional Andino Amazónico de Química de la Vida, Universidad Peruana Cayetano Heredia. Lima, Perú. \\ 2 Laboratorio Centinela de Helicobacter pylori, Instituto de Medicina Tropical Alexander von Humboldt, Universidad Peruana \\ Cayetano Heredia, Lima, Perú. \\ a Licenciada en Biología; ${ }^{b}$ biólogo, maestro en Bioquímica y Biología Molecular; ${ }^{c}$ químico farmacéutico, maestro en Bioquímica \\ y Biología Molecular, ${ }^{\mathrm{d}}$ químico farmacéutico, $\mathrm{PhD}$ en Farmacoquímica
}

\section{RESUMEN}

Objetivo. Analizar la susceptibilidad antimicrobiana de Helicobacter pylori a 5 antibióticos de referencia, en pacientes dispépticos del Servicio de Gastroenterología del Hospital Cayetano Heredia y la Clínica Cayetano Heredia en Lima, Perú. Materiales y métodos. Se colectaron biopsias gástricas de 500 pacientes diagnosticados con dispepsia. A partir de estas biopsias, se aislaron y cultivaron 273 cepas de H. pylori para confirmar la infección mediante el diagnóstico histológico y por cultivo. Finalmente, se analizó la susceptibilidad antimicrobiana mediante el método de microdilución en caldo y se evaluaron los perfiles de resistencia de cada antimicrobiano y los patrones de multirresistencia. Resultados. El diagnóstico de $H$. pylori por cultivo, comparado con la prueba histológica, reportó una sensibilidad del $83,8 \%$, una especificidad del $89,9 \%$ y un área bajo la curva de 0,87 (IC95\%: 0,84 a 0,90). La frecuencia de la infección en los servicios de gastroenterología del Hospital y la Clínica Cayetano Heredia fueron del 56,6\% (237/419) y 44,4\% (36/81), respectivamente. Según el Hospital/Clínica, se determinó la resistencia para amoxicilina $(45,1 \% / 29,6 \%)$, levofloxacino $(71,8 \% / 74,1 \%)$ y metronidazol $(69,8 \% / 63,0 \%)$. Los patrones de resistencia a múltiples antimicrobianos demostraron que las resistencias (dobles y triples) más frecuentes fueron con levofloxacino, metronidazol y amoxicilina. Conclusiones. La resistencia antimicrobiana de $H$. pylori ha aumentado con respecto a los años previos. Además, la resistencia múltiple de H. pylori presenta altas frecuencias en pacientes infectados. El método de microdilución en caldo podría ser implementado en los diferentes hospitales del Perú como una herramienta de vigilancia de la resistencia de H. pylori a los antimicrobianos.

Palabras clave: Helicobacter pylori; Prevalencia; Diagnóstico; Farmacorresistencia Bacteriana; Resistencia a Múltiples Medicamentos.

\section{DIAGNOSTIC PERFORMANCE OF THE CULTURE AND SUSCEPTIBILITY OF Helicobacter pylori IN PERUVIAN PATIENTS: RESULTS FROM A SENTINEL LABORATORY}

Citar como: Olano M, Chu M, Guzmán J, Castillo D, Sauvain M. Rendimiento diagnóstico del cultivo y susceptibilidad de Helicobacter pylori en pacientes peruanos: Resultados de un laboratorio centinela. Rev Peru Med Exp Salud Publica. 2021;38(3):40611. doi: https://doi.org/10.17843/ rpmesp.2021.383.7256

Correspondencia: Michel Henri Sauvain; michel.sauvain@ird.fr

Recibido: 02/02/2021

Aprobado: 08/09/2021

En línea: 31/09/2021

\section{ABSTRACT}

Objective: To analyze the antimicrobial susceptibility of Helicobacter pylori to 5 reference antibiotics, in a population of 500 dyspeptic patients from the Gastroenterology Service of the Hospital Cayetano Heredia $(\mathrm{n}=419)$ and the Clínica Cayetano Heredia $(\mathrm{n}=81)$ in Lima, Peru. Materials and methods: Gastric biopsies were collected from 500 patients diagnosed with dyspepsia. From these biopsies, 273 $H$. pylori strains were isolated and cultured to confirm $H$. pylori infection by histological and culture diagnosis. Finally, antimicrobial susceptibility was analyzed using the broth microdilution method, and the resistance profiles of each antimicrobial and multi-resistance patterns were evaluated by statistical analysis. Results: The diagnosis of $H$. pylori infection by culture, compared to histological testing, reported a sensitivity of $83.8 \%$, a specificity of $89.9 \%$ and an area under the curve (AUC) of 0.87 (95\% CI: 0.84 to 0.90 ). The frequency of infection in the gastroenterology services of the Hospital Cayetano Heredia and Clinic was 56.6\% (237/419) and 44.4\% (36/81), respectively. An increase in antimicrobial resistance to Amoxicillin (45.1\% / 29.6\%), Levofloxacin (71.8\%/ 74.1\%) and Metronidazole (69.8\% / $63.0 \%$ ) was found in the Hospital and the Clinic, respectively. Multiple resistance patterns showed that the most frequent resistance (double and triple) was to Levofloxacin, Metronidazole and Amoxicillin. Conclusions: The antimicrobial resistance of $H$. pylori has increased compared to that reported in previous years. Furthermore, H. pylori multiple resistance presents high frequencies in infected patients. The broth microdilution method could be implemented in different hospitals in Peru as a surveillance tool for $H$. pylori antimicrobial resistance.

Keywords: Helicobacter pylori; Prevalence; Diagnosis; Drug Resistance; Drug Resistance Multiple. 


\section{INTRODUCCIÓN}

Helicobacter pylori (H. pylori) es un patógeno humano, agente causal de enfermedades gastroduodenales asociadas a la malignidad del tejido estomacal ${ }^{(1)}$. Se estima que, en todo el mundo, la prevalencia de esta infección supera el 50\% y alcanza el $64 \%$ en América Latina. Esta prevalencia oscila ampliamente entre países; en el Perú, es del $45 \%$ y se observan prevalencias de hasta $80 \%$ en poblaciones de bajo nivel socioeconómico y acceso difícil a los servicios de salud ${ }^{(2)}$.

En la práctica clínica, la detección de $H$. pylori se efectúa a través de métodos invasivos o no invasivos. Los métodos no invasivos, como la prueba de urea espirada o la prueba de antígenos fecales, resultan de interés y muestran perfiles de sensibilidad y especificidad superiores al 95\%. De otro modo, los métodos invasivos, a partir de la toma de biopsia, facilitan, además, la posterior tipificación del patógeno. Según Frías ${ }^{(3)}$, la estrategia invasiva a partir de la toma de biopsia gástrica como estrategia de rutina permite una identificación histológica de la bacteria y su posterior aislamiento para realizar un análisis de interés epidemiológico ${ }^{(4)}$.

El tratamiento frente a la infección por $H$. pylori se basa en el empleo de la terapia triple estándar, la cual utiliza los antimicrobianos claritromicina más amoxicilina o metronidazol además de un inhibidor de bomba de protones. Ante el fracaso terapéutico de la infección, se recomienda el empleo de antimicrobianos de segunda línea, como el levofloxacino y la tetraciclina. Sin embargo, el incremento de la resistencia a los antimicrobianos es un problema de salud pública a nivel global y limita la utilidad del tratamiento por pérdida de eficacia como consecuencia del uso de dosis inadecuadas, la falta de adherencia al tratamiento, alta acidez gástrica, alta carga bacteriana $y$, como principal factor, la aparición de cepas resistentes a los antibióticos ${ }^{(5)}$.

El presente estudio evaluó la susceptibilidad a 5 antimicrobianos de referencia empleados en la primera y segunda línea de tratamiento frente a la infección por H. pylori, como son la amoxicilina, claritromicina, levofloxacino, metronidazol y la tetraciclina, a fin de determinar la frecuencia de resistencia. Además, se analizó la característica operativa del receptor (ROC, por sus siglas en inglés) para evaluar el desempeño del cultivo frente al diagnóstico histológico y se determinó la concordancia entre ellas empleando el índice kappa.

\section{MATERIALES Y MÉTODOS}

\section{Diseño y población de estudio}

Se realizó un estudio descriptivo de corte transversal, que incluyó a 500 voluntarios con síntomas de dispepsia que no habían recibido tratamiento para la infección por $H$. pylori tres meses antes de su enrolamiento y que presentaron in-

\section{MENSAJES CLAVE}

Motivación para realizar el estudio: En el Perú, se han incrementado las tasas de resistencia antimicrobiana a Helicobacter pylori. La información respecto a los fármacos de referencia aún es escasa debido a la dificultad del cultivo, lo que limita los ensayos de susceptibilidad antimicrobiana.

Principales hallazgos: Se muestra un elevado porcentaje de infección por $H$. pylori. La resistencia antimicrobiana se ha incrementado principalmente en los fármacos amoxicilina y levofloxacino.

Implicancias. El uso de la técnica de microdilución en caldo podría ser implementado en los diferentes hospitales del Perú como una herramienta de vigilancia de la resistencia de H. pylori a los antimicrobianos.

dicación para una toma de biopsia mediante un procedimiento endoscópico en el Servicio de Gastroenterología del Hospital Cayetano Heredia $(\mathrm{HCH})(\mathrm{n}=419)$ y la Clínica Cayetano Heredia $(\mathrm{CCH})(\mathrm{n}=81)$, Lima, Perú, entre marzo de 2016 y agosto de 2017. Los participantes firmaron de forma voluntaria el consentimiento informado del estudio aprobado por el Comité Institucional de Ética para Humanos de la Universidad Peruana Cayetano Heredia (Constancia 5902615 con código de inscripción 63157). Se codificaron cada muestra y cepa para mantener confidencialidad. Debido al diferente origen socioeconómico de los pacientes, el análisis fue independiente para cada centro ${ }^{(6)}$.

Para este estudio, se diagnosticó la infección por $H$. pylori utilizando el método invasivo endoscópico y se extrajeron las biopsias gástricas de los pacientes dispépticos para realizar el aislamiento de las cepas de $H$. pylori.

\section{Bacteria y condiciones de cultivo}

El aislamiento de las cepas de H. pylori se inició con la colecta de biopsias de mucosa gástrica mediante procedimiento endoscópico. Las biopsias fueron transportadas de forma independiente en caldo BHI (BD') suplementado con $10 \%$ de glicerol (Sigma-Aldrich ${ }^{\circ}$ a $4{ }^{\circ} \mathrm{C}$. Se disgregaron las muestras de mucosa gástrica empleando mallas de disgregación celular de $40 \mu \mathrm{m}\left(\mathrm{BD}^{*}\right)$; el proceso se desarrolló bajo condiciones de cadena frío en un intervalo de tiempo menor a tres horas y se tomó una alícuota del homogenizado para realizar el hemocultivo en agar (agar BHI $\left.\left[\mathrm{BD}^{\circ}\right]\right)$ con $10 \%$ de sangre desfibrinada de cordero, anfotericina B (Sigma-Alcrich') y suplemento selectivo Skirrow (Oxoid") a $37^{\circ} \mathrm{C}$ bajo condiciones de microaerobiosis ( $5 \%$ de $\mathrm{O}_{2}$ y $10 \% \mathrm{CO}_{2}$ ) durante 3-5 días ${ }^{(7)}$. 


\section{Diagnóstico histológico y por cultivo}

El análisis histológico se realizó mediante la tinción de las biopsias con hematoxilina-eosina y observación de las bacterias de $H$. pylori a $1000 \times$ en un microscopio óptico. El diagnóstico por cultivo se realizó mediante la identificación de colonias en las placas de agar sangre, según su apariencia, tinción Gram y las pruebas bioquímicas de oxidasa, catalasa y ureasa. Se consideró que el paciente estaba infectado cuando fue positivo para al menos una estrategia diagnóstica. Ambas estrategias fueron comparadas y se consideró el diagnóstico histológico como gold standard a fin de estimar el desempeño del diagnóstico por cultivo ${ }^{(7,8)}$.

\section{Evaluación de la susceptibilidad antimicrobiana}

Mediante la estrategia de microdilución en caldo se determinó la concentración mínima inhibitoria (CMI) y se empleó como control la cepa de referencia de Helicobacter pylori ATCC 43504. Para cada aislado se preparó un inóculo equivalente al estándar 2.0 McFarland $\left(1 \times 10^{7}\right.$ a $\left.1 \times 10^{8} \mathrm{UFC} / \mathrm{mL}\right)$ en caldo $\mathrm{BHI}\left(\mathrm{BD}^{\circ}\right)$ con $10 \%$ de suero fetal bovino (SigmaAldrich') y $1 \%$ de suplemento nutritivo para microorganismos fastidiosos (Isovitalex ${ }^{\circledR}$ ). Se agregaron amoxicilina, claritromicina, levofloxacino, metronidazol y tetraciclina (SigmaAldrich) al medio a las concentraciones iniciales de 0,48 $\mu \mathrm{g} / \mathrm{mL}, 2 \mu \mathrm{g} / \mathrm{mL}, 4 \mu \mathrm{g} / \mathrm{mL}$, $64 \mu \mathrm{g} / \mathrm{mL}$ y $4 \mu \mathrm{g} / \mathrm{mL}$ respectivamente, a partir de los cuales se realizaron cuatro diluciones seriadas. Enseguida, se incubó a $37^{\circ} \mathrm{C}$ bajo condiciones de microaerobiosis durante 72 horas. Las cepas aisladas se consideraron resistentes, tomando como referencia los puntos de corte recomendados por el Comité Europeo de Antibiogramas (EUCAST, por sus siglas en inglés) ${ }^{(9)}$. Los ensayos se realizaron por triplicado y se consideraron los perfiles de CMI recomendados por el Clinical and Laboratory Standards Institute $(\mathrm{CLSI})^{(10)}$ para la cepa de referencia ATCC 43504.

\section{Análisis estadístico}

Se realizó un análisis descriptivo de la frecuencia de casos para el perfil de resistencia de cada antimicrobiano y patrones de multirresistencia. El cálculo de sensibilidad, especificidad, valor predictivo positivo, valor predictivo negativo y área bajo la curva (ABC) se realizó mediante un análisis ROC, considerando gold standard la prueba histológica, y se determinó la concordancia entre ambas estrategias empleando el índice Kappa. El análisis de susceptibilidad fue reportado en frecuencias. Se calcularon la $\mathrm{CMI}_{50}$ y la $\mathrm{CMI}_{90}$ mediante un análisis de regresión Probit. El análisis se realizó con un nivel de confianza del 95\% y un nivel de significancia del 5\%. Se incorporaron los datos a una tabla de Excel 2016 y se analizaron con el paquete estadístico Stata/SE 15.1.

\section{RESULTADOS}

Se analizaron 500 muestras de pacientes con dispepsia. El diagnóstico por cultivo comparado a la prueba histológica presentó una sensibilidad de $83,8 \%$, una especificidad de 89,9\% y un ABC de 0,87 (IC95\%: 0,84 a 0,90) (Figura 1).

Las frecuencias de infección por $H$. pylori en los servicios de gastroenterología del Hospital y la Clínica Cayetano Heredia fueron de $56,6 \%$ y de $44,4 \%$, respectivamente, y se obtuvieron mediante el diagnóstico por cultivo microbiológico. Mientras que, mediante el método de diagnóstico histológico se obtuvieron las frecuencias de infección de $61,1 \%$ en el Hospital y de 56,8\% en la Clínica. La concordancia entre el diagnóstico por cultivo microbiológico y la histología determinada por el índice kappa $(\mathrm{K})$ fue de 0,71 para el Hospital y de 0,76 para la Clínica Cayetano Heredia (Tabla 1).

La evaluación de susceptibilidad en el Servicio de Gastroenterología del Hospital Cayetano Heredia, realizada mediante la prueba de microdilución en caldo, mostró frecuencias de resistencia antibiótica para $H$. pylori de $45,1 \%$ a la amoxicilina, de $33,3 \%$ a la claritromicina, de $71,8 \%$ al levofloxacino, de $69,8 \%$ al metronidazol y de $9,1 \%$ a la tetraciclina. En la Clínica Cayetano Heredia se observaron frecuencias de resistencia antibiótica de $29,6 \%$ a la amoxicilina, de $48,2 \%$ a la claritromicina, de $74,1 \%$ al levofloxacino y de $63,0 \%$ al metronidazol. La frecuencia de resistencia antibiótica a la tetraciclina en el Servicio de Gastroenterología de la Clínica Cayetano Heredia fue de $0 \%$. La concentración mínima que inhibe al 50\% $\left(\mathrm{CMI}_{50}\right)$ de las cepas de $H$. pylori para amoxicilina fue el doble en el Hospital $(0,27 \mu \mathrm{g} / \mathrm{ml})$ que lo observado en la Clínica Cayetano Heredia $(0.13 \mu \mathrm{g} / \mathrm{ml})$ (Tabla 2).

De los 179 casos analizados del Hospital Cayetano Heredia y de los 27 casos de la Clínica Cayetano Heredia positivos para H. pylori, la resistencia a dos antimicrobianos fue de $21,8 \%$ (39/179) y 33,3\% (9/27) respectivamente. Además, se observó que entre los aislados provenientes del Hospital Cayetano Heredia la resistencia triple presentó una mayor frecuencia $(29,1 \%)$, mientras que en la Clínica Cayetano Heredia la mayor frecuencia de casos multirresistentes correspondió a resistencia doble $(33,3 \%)$. El patrón de resistencia cuádruple de mayor

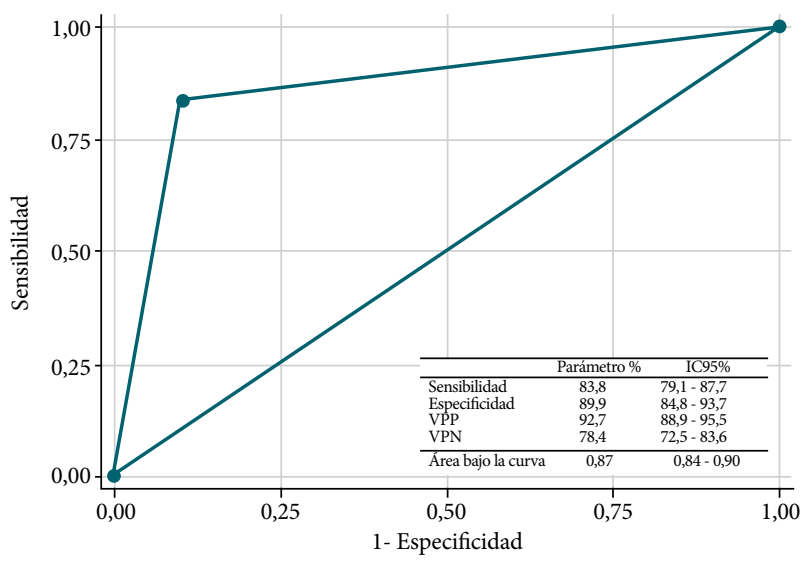

Figura 1. Desempeño de la prueba diagnóstica por cultivo comparado a la histología como prueba de referencia. 
Tabla 1. Distribución de los casos con dispepsia diagnosticados según la estrategia de diagnóstico por cultivo e histología

\begin{tabular}{|c|c|c|c|c|}
\hline \multirow{2}{*}{$\begin{array}{l}\text { Lugar de } \\
\text { estudio }\end{array}$} & \multicolumn{2}{|c|}{ Diagnóstico de H.pylori } & \multirow{2}{*}{ Kappa (IC95\%) } & \multirow{2}{*}{$\begin{array}{l}\text { Valor } \\
\text { de } p\end{array}$} \\
\hline & Histología (\%) & Cultivo (\%) & & \\
\hline $\begin{array}{l}\text { Hospital } \\
(\mathrm{n}=419)\end{array}$ & $61,1(256 / 419)$ & $56,6(237 / 419)$ & $0,71(0,64-0,79)$ & 0,001 \\
\hline $\begin{array}{l}\text { Clínica } \\
(\mathrm{n}=81)\end{array}$ & $56,8(46 / 81)$ & $44,4(36 / 81)$ & $0,76(0,57-0,94)$ & 0,001 \\
\hline
\end{tabular}

frecuencia en ambos centros involucró a los antimicrobianos amoxicilina, claritromicina, levofloxacino y metronidazol con porcentajes de 11,7\% y 11,1\%. La Clínica no presentó aislados con resistencia quíntuple, y entre los aislados provenientes del Hospital este patrón representa una frecuencia de 5,6\% (Tabla 3).

\section{DISCUSIÓN}

El diagnóstico de la infección por H. pylori por cultivo microbiológico se considera un método más específico de detección que permite desarrollar diversas estrategias, como la clasificación genotípica del microorganismo, la evaluación de la actividad y toxicidad de fármacos nuevos y usuales, la vigilancia de los perfiles de virulencia de las cepas de pacientes y la determinación de la resistencia a antimicrobianos, así como la implementación de biobancos que accedan a estudios epidemiológicos futuros (11). El presente estudio permitió determinar que el cultivo microbiológico muestra una moderada concordancia con las pruebas de detección de rutina observando indices kappa estadísticamente significativos de $\mathrm{K}=0,71$ para el Hospital y de K=0,76 para la Clínica Cayetano Heredia cuando se compara con la prueba histológica definida como gold standard.
Las frecuencias de infección por $H$. pylori fueron de $56,6 \%$ para el Hospital Cayetano Heredia y de $44,4 \%$ para la Clínica Cayetano Heredia. Estos patrones de frecuencia de infección son comparables a lo reportado en 2017 para el Hospital (58,9\%) y en 2015 para la Clínica Cayetano Heredia $(46,3 \%)^{(7,12,15)}$. En la actualidad, aún son insuficientes los estudios sobre frecuencias de infección por $H$. pylori en el país.

En el Perú, existen reportes sobre la resistencia de $H$. pylori a los antibióticos, empleando los métodos de microdilución en agar yema de huevo, difusión de disco, dilución en agar y epsilon test o E-test ${ }^{(13-15)}$. En 2019, Guzmán et al, reportaron tasas de resistencia antimicrobiana utilizando por primera vez el método de microdilución en caldo en cepas de $H$. pylori aisladas de pacientes dispépticos enrolados en 2015 para el mismo centro de salud privado. El porcentaje de infección por $H$. pylori fue del $46,3 \%$ y el porcentaje de resistencia a claritromicina fue del $52,3 \%$, obteniéndose así resultados contrastables con lo descrito en este trabajo.

Los complejos mecanismos moleculares de resistencia antibiótica en H. pylori limitan el empleo de estrategias moleculares para tipificar el conjunto de antimicrobianos de referencia empleados para su erradicación, por lo que las estrategias microbiológicas clásicas resultan idóneas para la evaluación de susceptibilidad antimicrobiana. En nuestro estudio, la evaluación de la susceptibilidad antimicrobiana mostró un aumento en las frecuencias de resistencia del metronidazol $69,8 \%(\mathrm{HCH}), 63 \%(\mathrm{CCH})$ respecto a $61,8 \%$ y $29,6 \%$ de 2017 y 2019 , respectivamente ${ }^{(15,12)}$; del levofloxacino, $71,8 \%(\mathrm{HCH})$ y $74,1 \%(\mathrm{CCH})$ superan la tasa de resistencia de $36,8 \%, 53,9 \%$ y $45,5 \%$ de 2011,2013 y 2015 , respectivamente $^{(16,15,12)}$; y de la claritromicina, $33,3 \%(\mathrm{HCH})$ y $48,2 \%(\mathrm{CCH})$, nuestros resultados fueron contrastables con lo reportado en 2017 por Boehnke ${ }^{(15)}$, Lima, Perú.

Con respecto al análisis de resistencia a la amoxicilina, hemos observado un incremento en ambos centros médicos

Tabla 2. Patrones de susceptibilidad de los aislados de H. pylori según el antimicrobiano de referencia utilizado

\begin{tabular}{lccccc}
\hline Variable & A & C & L & M & T \\
\hline H. pylori ATCC 43504 $(\mu \mathrm{g} / \mathrm{ml})$ & $0,015-0,125$ & $0,015-0,125$ & $0,064-0,5$ & $64-256$ & $0,12-1$ \\
Punto de corte (EUCAST) - Resistencia $(\mu \mathrm{g} / \mathrm{ml})$ & $>0,12$ & $>0,5$ & $>1$ & $>8$ & $>1$ \\
Frecuencia de resistencia - Hospital $(\%)$ & 45,1 & 33,3 & 71,8 & 69,8 & 9,1 \\
$\mathrm{CMI}_{50}$ & 0,27 & $<0,25$ & 2,40 & 17,12 & $<0,25$ \\
$\mathrm{CMI}_{50}$ & 0,91 & 3,11 & 4,62 & 35,55 & 1,80 \\
Frecuencia de resistencia - Clínica (\%) $_{\mathrm{CMI}_{50}}^{29,6}$ & 0,13 & 48,2 & 74,1 & 63,0 & 0,0 \\
$\mathrm{CMI}_{50}$ & 0,71 & $2,4,25$ & 7,41 & 22,35 & 0,25 \\
\hline
\end{tabular}

N: 273 cepas de Helicobacter pylori aislados (237 hospital / 36 clínica)

$\mathrm{CMI}_{50}$ : Concentración mínima que inhibe al 50\% de las cepas de H. pylori. $\mathrm{CMI}_{90}$ : Concentración mínima que inhibe al $90 \%$ de las cepas de $H$. pylori.

A: amoxicilina; C: claritromicina; L: levofloxacino; M: metronidazol; T: tetraciclina. 
Tabla 3. Patrones de resistencia múltiple de los aislados de H. pylori para cada antimicrobiano de referencia

\begin{tabular}{|c|c|c|c|c|c|c|c|c|c|c|c|c|c|c|}
\hline \multirow{2}{*}{$\begin{array}{l}\text { Lugar de } \\
\text { estudio }\end{array}$} & \multicolumn{5}{|c|}{ Resistencia doble } & \multicolumn{6}{|c|}{ Resistencia triple } & \multicolumn{2}{|c|}{$\begin{array}{l}\text { Resistencia } \\
\text { cuádruple }\end{array}$} & \multirow{2}{*}{$\begin{array}{c}\begin{array}{c}\text { Resistencia } \\
\text { a todos los } \\
\text { antibióticos }\end{array} \\
\text { ACLMT }\end{array}$} \\
\hline & $\mathbf{A L}$ & $\mathrm{AM}$ & CL & CM & LM & ACL & ACM & ALM & CLM & CLT & LMT & ACLM & ALMT & \\
\hline Hospital n (\%) & $6(3,4)$ & $7(3,9)$ & $3(1,7)$ & $2(1,1)$ & $21(11,7)$ & $3(1,7)$ & $4(2,2)$ & $26(14,5)$ & $15(8,4)$ & $1(0,6)$ & $3(3,7)$ & $21(11,7)$ & $2(1,1)$ & $10(5,6)$ \\
\hline Total n (\%) & $39(21,8)$ & & & & & $52(29,1)$ & & & & & & $23(12,8)$ & & $10(5,6)$ \\
\hline Clínica n (\%) & $1(3,7)$ & -- & $1(3,7)$ & -- & $7(25,9)$ & $1(3,7)$ & $1(3,7)$ & $1(3,7)$ & $5(18,5)$ & -- & -- & $3(11,1)$ & -- & -- \\
\hline Total n (\%) & $9(33,3)$ & & & & & $8(29,6)$ & & & & & & $3(11,1)$ & & \\
\hline
\end{tabular}

$\mathrm{N}$ : 206 cepas de Helicobacter pylori multirresistentes (179 hospital / 27 clínica)

A: amoxicilina; C: claritromicina; L: levofloxacino; M: metronidazol; T: tetraciclina.

(45,1\% para el Hospital y $29,6 \%$ para la Clínica) comparado a otros autores como Boehnke et al, (32,9\% en Hospital) ${ }^{(15)}$ y Guzman et al, (4,6\% en Clínica) ${ }^{(12)}$. El aumento de la frecuencia de resistencia puede estar influenciado por el incremento del consumo de estos fármacos en los últimos años, llegando al $70 \%$ respecto a otros antimicrobianos y su consumo sin prescripción médica ${ }^{(17)}$.

En el presente estudio, el 69,3\% y el 74,1\% de los aislamientos provenientes de pacientes dispépticos del Hospital y la Clínica Cayetano Heredia presentaron un patrón de multirresistencia. La resistencia triple $(29,1 \%)$ y la doble $(33,3 \%)$ presentaron las mayores frecuencias para el Hospital Cayetano Heredia y la Clínica Cayetano Heredia, respectivamente. Solo para el Hospital se presentó una multirresistencia a los 5 antimicrobianos (5,6\%). Además, se observó que el fármaco menos afectado fue la tetraciclina. Esta multirresistencia es mayor en comparación con la obtenida en otros países. En México, informaron resistencia doble (30,7\%) y resistencia triple $(8,7 \%)$ entre los aislados de H. pylori ${ }^{(18)}$. Del mismo modo, se detectaron múltiples cepas resistentes en pacientes asiáticos $(8,3 \%)$, estadounidenses $(15 \%)$ y europeos $(8,9 \%)^{(19,20)}$.

En la actualidad, la terapia triple, que incluye dos antibióticos y un inhibidor de la bomba de protones, se utiliza como tratamiento para la infección por $H$. pylori. Los antibióticos más utilizados son la claritromicina, el metronidazol, la amoxicilina y el levofloxacino, este último como segunda línea o fármaco de rescate. En nuestro estudio, se observó que los patrones de multirresistencia se distribuyen de manera uniforme para ambos centros, con una mayor frecuencia del patrón de resistencia doble metronidazol y levofloxacino de $11,7 \%$ y $25,9 \%$ para los aislamientos del Hospital Cayetano Heredia y de la Clínica Cayetano Heredia, respectivamente. Además, los patrones de resistencia triple principalmente observados involucran tanto a metronidazol como a levofloxacino y adicionan amoxicilina entre los aislados de Hospital (14,5\%), mientras que en la Clínica el patrón triple adiciona a la asociación metronidazolylevofloxacino el fármaco claritromicina con una tasa de resistencia triple de 18,5\%. La mayor frecuencia de casos con resistencia cuádruple tanto en el Hospital como en la Clínica considera a los fármacos amoxicilina, claritromicina, levofloxacino y metronidazol. A pesar de la baja frecuencia del patrón de resistencia múltiple, la amoxicilina, claritromicina y tetraciclina, con un inhibidor de la bomba de protones podrían sugerirse como terapia combinada para el tratamiento de la infección por $H$. pylori en nuestra población. Sin embargo, se debe considerar la alta frecuencia de resistencia detectada de manera independiente a la amoxicilina y claritromicina ( $45,1 \%$ y $33,3 \%$, respectivamente).

La obtención de los resultados de susceptibilidad antimicrobiana, mediante el método de microdilución en caldo, se basó en la determinación de la CMI y los puntos de cortes que reporta la EUCAST para indicar si la cepa de $H$. pylori es sensible o resistente a los antibióticos en estudio. Se necesitan más estudios que validen el método de microdilución en caldo como un método estándar para la evaluación de la resistencia de H. pylori frente a los antimicrobianos. También es importante implementar estudios de carácter longitudinal para la evaluación de la eficacia de los tratamientos actuales contra la infección y, de esta manera, proponer una vigilancia de rutina a la resistencia antimicrobiana de $H$. pylori en el país.

En conclusión, la frecuencias de resistencia antibiótica para H. pylori en el Hospital Cayetano Heredia fue de $45,1 \%$ para amoxicilina, 33,3\% a la claritromicina, $71,8 \%$ al levofloxacino, $69,8 \%$ al metronidazol y $9,1 \%$ a la tetraciclina. En la Clínica Cayetano Heredia se observó frecuencias de resistencia de 29,6\% a la amoxicilina, $48,2 \%$ a la claritromicina, $74,1 \%$ al levofloxacino y de $63,0 \%$ al metronidazol.

Agradecimientos: Agradecemos al Dr. Jorge Luis Huerta-Mercado Tenorio y al personal de los Servicios de Gastroenterología del Hospital y de la Clínica Cayetano Heredia, especialmente a la enfermera Nátaly Ojeda Oyague, por su interés y dedicación a nuestro trabajo de investigación. 
Contribución de los autores: JG, DC, y MS concibieron y diseñaron el estudio; JG, MC y MO recopilaron los datos; JG y MO procesaron, analizaron e interpretaron los datos; JG y MO redactaron del artículo; DC y MS revisaron críticamente el artículo. Todos los autores aprobaron la versión final.

Financiamiento: FONDECYT y CIENCIACTIVA, a través del apoyo financiero recibido en el marco del proyecto «Prototipo de

\section{REFERENCIAS BIBLIOGRÁFICAS}

1. Dubois A. Spiral bacteria in the human stomach: the gastric helicobacters. Emerg Infect Dis. 1995;1(3):79-85. https://www.ncbi.nlm.nih.gov/pmc/ articles/PMC2626874/.

2. Sjomina O, Pavlova J, Niv Y, Leja M. Epidemiology of Helicobacter pylori infection. Helicobacter. 2018;23 Suppl 1: e12514. doi: 10.1111/hel.12514.

3. Frías-Ordoñez JS, Otero-Regino W. Aspectos prácticos en métodos diagnósticos para la infección por Helicobacter pylori: una revisión narrativa. Rev Gastroenterol Perú. 2017; 37(3): 246-253.

4. Lash JG, Genta RM. Adherence to the Sydney System guidelines increases the detection of Helicobacter gastritis and intestinal metaplasia in 400738 sets of gastric biopsies. Aliment Pharmacol Ther. 2013 Aug;38(4):424-31. doi: 10.1111/apt.12383.

5. Malfertheiner P, Megraud F, O'Morain CA. on behalf of the European Helicobacter and Microbiota Study Group and Consensus panel, et al. Management of Helicobacter pylori infection-the Maastricht V/Florence Consensus Report Gut 2017;66:6-30. doi: 10.1136/gutjnl-2016-312288.

6. Aliaga RJ, Cedron CH, Pinto VJ. Comparación de prevalencia de infección por Helicobacter pylori en pacientes con dispepsia entre dos instituciones de diferentes estratos socioeconómicos en el periodo 2017-2018. Rev. Gastroenterol Perú. 2019;39(3):211-214.

7. Guzmán J, Castillo D, Ojeda M, Sauvain M. Susceptibilidad antimicrobiana y mutaciones en el gen ARNr 23s de Helicobacter pylori en pacientes dispépticos. Rev Peru Med Exp Salud Pública. 2019;36(2):270-4. doi: http://dx.doi.org/10.17843/rpmesp.2019.362.3901.

8. Ndip RN, MacKay WG, Farthing MJ, Weaver LT. Culturing Helicobacter pylori from clinical specimens: review of microbiologic methods. J Pediatr Gastroenterol Nutr. 2003;36(5):616-22. doi: 10.1097/00005176200305000-00005.

9. European Committee on Antimicrobial Susceptibility Testing. Breakpoint tables for interpretation of MICs and zone diameters. Version 9.0, 2019 [Internet]. Basilea: EUCAST; 2019 [Citado el 20 de octubre de 2018] Disponible en: http://www.eucast.org/fileadmin/src/media/PDFs/EUCAST_files/Breakpoint_tables/v_9.0_Breakpoint_Tables.pdf.

10. Clinical and Laboratory Standars Institute. Methods for antimicrobial dilution and disk susceptibility testing of infrequently isolated or fastidious bacteria; Approved Guideline-Second edition. Wayne, PA: CLSI; 2010. [Citado 20 octubre de 2018] Disponible en: https://clsi.org/media/1450/ m45ed3_sample.pdf.

11. Frías-Ordoñez JS, Otero-Regino W. Aspectos prácticos en métodos diagnósticos para la infección por Helicobacter pylori: una revisión narrativa. Rev Gastroenterol Perú. 2017; 37(3): 246-253. laboratorio centinela para controlar la resistencia de Helicobacter pylori a los antibióticos en el Perú». Proyecto 1172015 ejecutado en el Instituto de Medicina Tropical Alexander von Humboldt - UPCH con el Instituto de Investigación para el Desarrollo IRD-Francia.

Conflicto de interés: Los autores declaramos no tener conflicto de interés.

12. Guzmán J, Castillo D, Ojeda M, Sauvain M. Susceptibilidad an timicrobiana y mutaciones en el gen ARNr 23s de Helicobacter pylori en pacientes dispépticos. Rev Peru Med Exp Salud Pública. 2019;36(2):270-4. doi: 10.17843/rpmesp.2019.362.3901.

13. Vásquez A, Valdez Y, Gilman RH, McDonald JJ, Westblom TU, Berg D, et al. Metronidazole and clarithromycin resistance in Helicobacter pylori determined by measuring MICs of antimicrobial agents in color indicator egg yolk agar in a miniwell format. The Gastrointestinal Physiology Working Group of Universidad Peruana Cayetano Heredia and the Johns Hopkins University. J Clin Microbiol. 1996;34(5):1232-1234. doi: 10.1128/jcm.34.5.1232-1234.1996.

14. Alarcón T, Domingo D, López-Brea M. Los problemas de resistencia a antibióticos con Helicobacter pylori. Int J Antimicrob Agents. 1999; 12(1):19-26. doi: 10.1016/s0924-8579(99)00051-5.

15. Boehnke KF, Valdivieso M, Bussalleu A, Sexton R, Thompson KC, Osorio $\mathrm{S}$, et al. Antibiotic resistance among Helicobacter pylori clinical isolates in Lima, Peru. Infect Drug Resist. 2017; 10:85-90. doi 10.2147/IDR.S123798.

16. Mochizuki H, Noriega AP. Determinación de la susceptibilidad de cepas de Helicobacter pylori a Levofloxacino en formato pequeño y método de difusión en disco usando Agar yema de huevo. Rev Gastroenterol Perú. 2011;31(3):224-9.

17. Megraud F, Coenen S, Versporten A, Kist M, Lopez-Brea M, Hirschl $\mathrm{AM}$, et al. Helicobacter pylori resistance to antibiotics in Europe and its relationship to antibiotic consumption. Gut. 2013;62(1):34-42. doi: 10.1136/gutjnl-2012-302254.

18. Torres J, Camorlinga-Ponce M, Perez-Perez G, Medrazo-De la Garza A, Dehesa M, Gonzalez-Valencia G, et al. Increasing multidrug resistance in Helicobacter pylori strains isolated from children and adults in Mexico. J Clin Microbiol. 2001; 39:2677-80. doi: 10.1128/ JCM.39.7.2677-2680.2001.

19. De Francesco V, Floriana G, Cesare H, Gianpiero M, Lucy V, Carmine P, et al. Worldwide H. pylori antibiotic resistance: a systematic review. J Gastrointestin Liver Dis. 2010;19(4):409-14.

20. Kouitcheu Mabeku LB, Eyoum Bille B, Tepap Zemnou C. Broad spectrum resistance in Helicobacter pylori isolated from gastric biopsies of patients with dyspepsia in Cameroon and efflux-mediated multiresistance detection in MDR isolates. BMC Infect Dis 19, 880 2019. doi: 10.1186/s12879-019-4536-8. 